\title{
The Markovian Regime-Switching Risk Model with Constant Dividend Barrier under Absolute Ruin
}

\author{
Wenguang $\mathrm{Yu}^{1}$, Yujuan Huang ${ }^{2}$ \\ ${ }^{1}$ School of Statistics and Mathematics, Shandong Economic University, Jinan, China \\ ${ }^{2}$ Department of Mathematics and Physics, Shandong Jiaotong University, Jinan, China \\ E-mail:yuwg@mail.sdu.edu.cn \\ Received July 26, 2011; revised September 8, 2011; accepted September 22, 2011
}

\begin{abstract}
In this paper, we consider the dividend payments prior to absolute ruin in a Markovian regime-switching risk process in which the rate for the Poisson claim arrivals and the distribution of the claim amounts are driven by an underlying Markov jump process. A system of integro-differential equations with boundary conditions satisfied by the moment-generating function, the $n$th moment of the discounted dividend payments prior to absolute ruin and the expected discounted penalty function, given the initial environment state, are derived. Then, the matrix form of systems of integro-differential equations satisfied by the discounted penalty function are presented. Finally, we obtain the integro-differential equations satisfied by the time to reach the dividend barrier.
\end{abstract}

Keywords: Absolute Ruin, Debit Interest, Moment-Generating Function, Markovian Regime-Switching Risk Model, Dividend Barrier, Integro-Differential Equation

\section{Introduction}

In recent years, ruin theory under regime-switching model is becoming a popular topic. This model is proposed in Reinhard [1] and Asmussen [2]. Asmussen calls it a Markov-modulated risk model. The purpose for this generalization is to enhance the flexibility of the model parameter settings for the classical risk process. This model can capture the feature that insurance policies may need to change if economical or political environment changes. There are many papers published on ruin probabilities and the related problems under the Markov regime-switching risk model. For example, Lu and Li [3] study ruin probabilities under this model. Ng and Yang [4] obtain an upper bound for the joint distribution of surplus before and at ruin under the regime-switching model by using a martingale approach. Ng and Yang [5] present some explicit results for the joint distribution of surplus before and at ruin under this model in the cases of zero initial surplus and phase type claim size distributions, respectively. $\mathrm{Li}$ and Lu [6] investigate the moments of the dividend payments and related problems in a Markov-modulated risk model. Lu and Li [7] and Liu et al. [8] consider a regimeswitching risk model with a threshold dividend strategy. Zhu and Yang [9] study a more general Markovian regime-switching risk model in which the premium, the claim intensity, the claim amount, the dividend payment rate and the dividend threshold level are influenced by an external Markovian environment process. Wei et al. [10] consider the Markov-modulated insurance risk model with tax. However, there is no work that deals with the absolute ruin in a regime-switching risk model. This motivates us to investigate such a risk model in this work.

Due to its practical importance, the issue of absolute ruin problem has received attention in risk theory. Zhou and Zhang [11] got the explicit expression of the absolute ruin probability for the classical risk model with exponential individual claim by using the Markov property. Cai [12] defined Gerber-Shiu function at absolute ruin and derived a system of the integro-differential equations satisfied by the Gerber-Shiu function. Yuan and Hu [13] investigate the absolute ruin in the compound Poisson risk model with nonnegative interest and a constant dividend barrier. Wang and Yin [14] studied the dividend payments in the classical risk model under absolute ruin with debit interest. Wang et al. [15] considered the dividend payments in a compound Poisson risk model with credit and debit interest under absolute ruin.

Now denote by $\{J(t) ; t \geq 0\}$ the external environment process, and suppose that it is a homogeneous, irreducible and recurrent Markov process with a finite state space 
$E=\{1,2,3, \cdots, m\}$ and intensity matrix $\Lambda=\left\{\alpha_{i j}\right\}_{i, j=1}^{m}$, where $\alpha_{i i}=-\alpha_{i}$ for $i \in E$. Let $N(t)$ be the number of claims occurring in $(0, t]$. If $J(s)=i$ for all $s$ in a small interval $(t, t+h]$, then the number of claims occurring in that interval, $N(t+h)-N(t)$, is assumed to follow a Poisson distribution with parameter $\lambda(>0)$, and the $n$ th claim amounts $X_{n}$ have distribution $F(x)$ with density function $f_{i}(x)$ and finite mean $u_{i}(i \in E)$. Moreover, We assume that the process $\{J(t) ; t \geq 0\}$ and the process $\{N(t) ; t \geq 0\}$ has independent increments. Then

$$
\operatorname{Pr}[N(t+h)=n+1 \mid N(t)=n, J(s)=i,
$$

for $t<s \leq t+h]=\lambda_{i} h+o(h)$.

The process $\{N(t) ; t \geq 0\}$ is called a Markov-modu lated Poisson process, which is a special case of Cox processes. It also can be seen as a Poisson process with the parameter driven by an external environment process $\{J(t) ; t \geq 0\}$.

In this paper, we consider a regime-switching risk model with debit interest and constant dividend barrier. In this model, the insurer could borrow an amount of money equal to the deficit at a debit interest force $\beta$ when the surplus is negative. Meanwhile, the insurer will repay the debts continuously from his premium income. The negative surplus may return to a positive level. However, when the negative surplus attains the level $-c / \beta$ or is below $-c / \beta$, the surplus is no longer able to be positive, because the debts of the insurer at this time are greater than or equal to $c / \beta$, which is the present value at that time for all premium income available after that point. Absolute ruin occurs at this moment. Moreover, When the surplus exceeds the constant barrier $b(\geq u)$, dividends are paid continuously so the surplus stays at the level $b$ until a new claim occurs. The corresponding sur- plus process $\left\{U_{b}(t) ; t \geq 0\right\}$ is given by

$$
\begin{aligned}
\mathrm{d} U_{b}(t)= & {[c+\beta U(t) I(U(t)<0)] \mathrm{d} t } \\
& -\mathrm{d}\left(\sum_{k=1}^{N(t)} X_{k}\right)
\end{aligned}
$$

where $U(0)=u$ is the initial surplus and $I(B)$ means the indicator function of an event $B$.

Let $D(t)$ be the cumulative amount of dividends paid out up to time $t$ and $\delta>0$ the force of interest, then

$$
D_{u, b}=\int_{0}^{T_{b}} \mathrm{e}^{-\delta t} \mathrm{~d} D(t)
$$

is the present value of all dividends until time of ruin $T_{b}$, where $T_{b}$ denoted by $T_{b}=\inf \left\{t \geq 0: U_{b}(t) \leq-c / \beta\right\}$ is the time of absolute ruin.

In the sequel we will be interested in the momentgenerating function $M_{i}(u, y, b)=E\left[\mathrm{e}^{y D_{u, b}} \mid J(0)=i\right], i \in E$, and the $n$th moment function

$$
\begin{array}{r}
V_{n, i}(u ; b)=E\left[D_{u, b}^{n} \mid J(0)=i\right], \\
n \in N, i \in E
\end{array}
$$

with $V_{0, i}(u ; b)=1$, and the expected discounted penalty function, for $i \in E$

$$
\begin{aligned}
& \Phi_{i}(u, b) \\
= & E\left[\mathrm{e}^{-\delta T_{b}} \omega\left(U_{b}\left(T_{b}-\right),\left|U_{b}\left(T_{b}\right)\right|\right) \times I\left(T_{b}<\infty\right) \mid U_{b}(0)=u, J(0)=i\right]
\end{aligned}
$$

where, $U_{b}\left(T_{b}-\right)$ is the surplus prior to absolute ruin and $\left|U_{b}\left(T_{b}\right)\right|$ is the deficit at absolute ruin. The penalty function $\omega\left(x_{1}, x_{1}\right)$ is an arbitrary nonnegative measure able function defined on $(-c / \beta,+\infty) \times(c / \beta,+\infty)$. Throughout this paper we assume that $M_{i}(u, y, b), V_{n, i}(u ; b)$ and $\Phi_{i}(u, b)$ are sufficiently smooth functions in $u$ and $y$, respectively.

Then the expected present value of the total dividend payments until ruin in the stationary case is given by

$$
V(u, b)=\sum_{i=1}^{m} \pi_{i} V_{i}(u, b)
$$

where $\pi=\left(\pi_{1}, \cdots, \pi_{m}\right)$ is the stationary initial distribution of process $\{J(t) ; t \geq 0\}$.

The rest of the paper is organized as follows. In Section 2, we obtain the integro-differential equations for the moment-generating function and boundary conditions in a regime-switching risk model. In Section 3, the integrodifferential equations satisfied by higher moment of the dividend payments and boundary conditions are derived. In the last section, we get the systems of integro-differential equations for $\Phi_{i}(u, b)$ and it's matrix form.

\section{Moment-Generating Function of $D_{u . b}$}

We now derive the systems of integro-differential equations satisfied by $M_{i}(u, y, b)$, for $i \in E$. Clearly, the moment-generating function $M_{i}(u, y ; b)$ behaves differently, depending on whether its initial surplus $u$ is below zero or above the barrier level $b$. Hence, we write $M_{1 i}(u, y ; b)$ for $0 \leq u<b$ and $M_{2 i}(u, y ; b)$ for $-c / \beta<u \leq 0$.

Theorem 2.1

For $0 \leq u \leq b$, 


$$
\begin{aligned}
c \frac{\partial M_{1 i}(u, y ; b)}{\partial u}= & \delta y \frac{\partial M_{1 i}(u, y ; b)}{\partial y}+\lambda_{i} \partial M_{1 i}(u, y ; b) \\
& -\lambda_{i} \int_{0}^{u} M_{1 i}(u-x, y ; b) \mathrm{d} F_{i}(x) \\
& -\lambda_{i} \int_{0}^{u+\frac{c}{\beta}} M_{2 i}(u-x, y ; b) \mathrm{d} F_{i}(x) \\
& -\lambda_{i} \bar{F}_{i}(u+c / \beta)-\sum_{k=1}^{m} \alpha_{i k} M_{1 k}(u, y ; b), \\
& i \in E
\end{aligned}
$$

and, for $-c / \beta<u<0$,

$$
\begin{aligned}
(\beta u+c) \frac{\partial M_{2 i}(u, y ; b)}{\partial u}= & \delta y \frac{\partial M_{2 i}(u, y ; b)}{\partial y} \\
& +\lambda_{i} M_{2 i}(u, y ; b)-\lambda_{i} \bar{F}_{i}(u+c / \beta) \\
& -\lambda_{i} \int_{0}^{u+\frac{c}{\beta}} M_{2 i}(u-x, y ; b) d F_{i}(x) \\
& -\sum_{k=1}^{m} \alpha_{i k} M_{2 k}(u, y ; b) \\
& i \in E
\end{aligned}
$$

with boundary conditions, for $i \in E$,

$$
\begin{gathered}
\left.\frac{\partial M_{1 i}(u, y ; b)}{\partial u}\right|_{u=b}=y M_{1 i}(b, y ; b) \\
M_{2 i}(-c / \beta, y ; b)=1
\end{gathered}
$$

Proof. Considering a small time interval $[0, t]$, with $t(t>0)$ being sufficiently small that $u+c t<b$, there are four possible events regarding the occurrence of the claim and the change of the environment:

1) No claim and no change of environment occur in $[0, t]$;

2) A claim occurs in $[0, t]$ (it can either cause the absolute ruin or not);

3) The environment changes in $[0, t]$;

4) Two or more events occur in $[0, t]$.

In view of the strong Markov property of the surplus process $\left\{U_{b}(t), t \geq 0\right\}$, we have

$$
M_{i}(u, y ; b)=E\left[M_{i} U_{b}(t), y \mathrm{e}^{-\alpha t} ; b\right] .
$$

Conditioning on the event occurring in the interval $[0, t]$, we have

$$
\begin{aligned}
M_{1 i}(u, y ; b)= & \left(1-\alpha_{i} t-\lambda_{i} t\right) M_{1 i}\left(u+c t, y \mathrm{e}^{-\delta t} ; b\right) \\
& +\lambda_{i} t \int_{0}^{u+c t} M_{1 i}\left(u+c t-x, y \mathrm{e}^{-\delta t} ; b\right) \mathrm{d} F_{i}(x) \\
& +\lambda_{i} t \int_{u+c t}^{u+c t+\frac{c}{\beta}} M_{2 i}\left(u+c t-x, y \mathrm{e}^{-\delta t} ; b\right) \mathrm{d} F_{i}(x) \\
& +\lambda_{i} t \bar{F}_{i}(u+c t+c / \beta) \\
& +t \sum_{k=1, k \neq i}^{m} \alpha_{i k} M_{1 k}\left(u+c t, y \mathrm{e}^{-\delta t} ; b\right)+o(t) .
\end{aligned}
$$

Taylor's expansion gives

$$
\begin{aligned}
M_{1 i}\left(u+c t, y e^{-\delta t} ; b\right)= & M_{1 i}(u, y ; b) \\
& +c t \frac{\partial M_{1 i}(u, y ; b)}{\partial u} \\
& -\delta y t \frac{\partial M_{1 i}(u, y ; b)}{\partial u} \\
& +o(t) .
\end{aligned}
$$

Substituting (2.7) into (2.6), dividing both sides by $t$, and letting $t \rightarrow 0$, we obtain (2.1).

Similarly, when $-c / \beta<u \leq 0$, we still consider a small time interval $[0, t]$, with $t(t>0)$ being sufficiently small so that the surplus will not reach 0 in the time interval. Let $t_{0}$ be the solution to

$$
h_{\beta}(u, t)=u \mathrm{e}^{\beta t}+c\left(\mathrm{e}^{\beta t}-1\right) / \beta=0
$$

then $h_{\beta}(u, t)$ is the surplus at time $t \leq t_{0}$ if no claim occurs prior to time $t_{0}$. We assume $t \leq t_{0}$. So conditioning on the time and the amount of the first claim, we have

$$
\begin{aligned}
M_{2 i}(u, y ; b)= & \left(1-\alpha_{i} t-\lambda_{i} t\right) M_{2 i}\left(h_{\beta}(u, t), y \mathrm{e}^{-\delta t} ; b\right) \\
& +\lambda_{i} t \int_{0}^{h_{\beta}(u, t)+\frac{c}{\beta}} M_{2 i}\left(h_{\beta}(u, t)-x, y \mathrm{e}^{-\delta t} ; b\right) \mathrm{d} F_{i}(x) \\
& +\lambda_{i} t \bar{F}_{i}\left(h_{\beta}(u, t)+c / \beta\right) \\
& +t \sum_{k=1, k \neq i}^{m} \alpha_{i k} M_{2 k}\left(h_{\beta}(u, t), y \mathrm{e}^{-\delta t} ; b\right)+o(t)
\end{aligned}
$$

By Taylor's expansion

$$
\begin{aligned}
M_{2 i}\left(h_{\beta}(u, t), y \mathrm{e}^{-\delta t} ; b\right)= & M_{2 i}(u, y ; b) \\
& +(\beta u+c) t \frac{\partial M_{2 i}(u, y ; b)}{\partial u} \\
& -\delta y t \frac{\partial M_{2 i}(u, y ; b)}{\partial y}+o(t)
\end{aligned}
$$


Substituting (2.9) into (2.8), dividing both sides by $t$, and letting $t \rightarrow 0$, we obtain (2.2).

When the initial surplus is $b$, we obtain

$$
\begin{aligned}
M_{1 i}(b, y ; b)= & \left(1-\alpha_{i} t-\lambda_{i} t\right) \mathrm{e}^{y c t} M_{1 i}\left(b, y \mathrm{e}^{-\delta t} ; b\right) \\
& +\lambda_{i} t e^{y c t} \int_{0}^{b} M_{1 i}\left(b-x, y \mathrm{e}^{-\delta t} ; b\right) \mathrm{d} F_{i}(x) \\
& +\lambda_{i} t e^{y c t} \int_{b}^{b+\frac{c}{\beta}} M_{2 i}\left(b-x, y \mathrm{e}^{-\alpha t} ; b\right) \mathrm{d} F_{i}(x) \\
& +\lambda_{i} t \mathrm{e}^{y c t} \frac{1}{F_{i}}(b+c / \beta) \\
& +t \mathrm{e}^{y c t} \sum_{k=1, k \neq i}^{m} \alpha_{i k} M_{1 k}\left(b, y \mathrm{e}^{-\delta t} ; b\right)+o(t)
\end{aligned}
$$

Using Taylor's expansion and noting that $\alpha_{i i}=-\alpha$, we have, for $i \in E$,

$$
\begin{aligned}
\delta y \frac{\partial M_{1 i}(b, y ; b)}{\partial y} & +\left(\lambda_{i}-c y\right) M_{1 i}(b, y ; b) \\
= & \lambda_{i} \int_{0}^{b} M_{1 i}\left(b-x, y \mathrm{e}^{-\delta t} ; b\right) \mathrm{d} F_{i}(x) \\
& +\lambda_{i} \int_{b}^{b+\frac{c}{\beta}} M_{2 i}\left(b-x, y \mathrm{e}^{-\delta t} ; b\right) \mathrm{d} F_{i}(x) \\
& +\lambda_{i} \overline{F_{i}}(b+c / \beta) \\
& +\sum_{k=1, k \neq i}^{m} \alpha_{i k} M_{1 k}\left(b, y \mathrm{e}^{-\delta t} ; b\right)+o(t)
\end{aligned}
$$

Letting $u \uparrow b$ in (2.1) and comparing it with (2.11), we obtain (2.3).

When $u=-c / \beta$, absolute ruin is immediate. Thus, no dividend is paid. So we obtain (2.4). Theorem 2.1 is proved.

Theorem 2.2 For $i \in E$,

$$
M_{1 i}(0+, y ; b)=M_{2 i}(0-, y ; b)
$$

Proof. For $-c / \beta<u \leq 0$, letting $\tau_{0}$ be the time that the surplus reach 0 for the first time from $u<0$ and using the Markov property of the surplus process, we obtain

$$
\begin{aligned}
M_{2 i}(u, y ; b)= & E_{i}^{u}\left[I\left(\tau_{0}<T_{b}\right) \mathrm{e}^{y D_{u, b}}\right] \\
& +E_{i}^{u}\left[I\left(\tau_{0} \geq T_{b}\right) \mathrm{e}^{y D_{u, b}}\right] \\
= & E_{i}^{u}\left[I\left(\tau_{0}<T_{b}\right) \exp \left\{y \int_{0}^{T_{b}-\tau_{0}} \mathrm{e}^{-\delta t} \mathrm{~d} D\left(t+\tau_{0}\right)\right\}\right] \\
& +P\left(\tau_{0} \geq T_{b}\right) \\
= & E_{i}^{u}\left[I\left(\tau_{0}<T_{b}\right) \exp \left\{y \mathrm{e}^{-\delta \tau_{0}} \int_{\tau_{0}}^{T_{b}} \mathrm{e}^{-\delta t} \mathrm{~d} D(t)\right\}\right] \\
& +P\left(\tau_{0} \geq T_{b}\right) \\
\leq & M_{1 i}(0, y ; b)+P\left(\tau_{0} \geq T_{b}\right)
\end{aligned}
$$

Similarly, we obtain

$$
\begin{aligned}
M_{2 i}(u, y ; b) \geq & E_{i}^{u}\left[I\left(\tau_{0}<T_{b}, \tau_{0}=t_{0}\right) \mathrm{e}^{y D_{u, b}}\right] \\
& +E_{i}^{u}\left[I\left(\tau_{0} \geq T_{b}\right) \mathrm{e}^{y D_{u, b}}\right] \\
= & M_{1 i}(0, y ; b) E_{i}^{u}\left[\mathrm{e}^{-\delta \tau_{0}} I\left(\tau_{0}<T_{b}, \tau_{0}=t_{0}\right)\right] \\
& +P\left(\tau_{0} \geq T_{b}\right) \\
= & M_{1 i}(0, y ; b) \mathrm{e}^{-\delta \tau_{0}} P\left(T_{1}>t_{0}\right)+P\left(\tau_{0} \geq T_{b}\right) \\
= & \mathrm{e}^{-\left(\lambda_{i}+\delta\right) t_{0}} M_{1 i}(0, y ; b)+P\left(\tau_{0} \geq T_{b}\right)
\end{aligned}
$$

where $T_{1}$ is the time of the first claim.

When $u \uparrow 0$, we notice that $\tau_{0}$ and $t_{0}$ both go into zero. Letting $u \uparrow 0$ in (2.13) and (2.14) and in view of

$$
\lim _{u \uparrow 0} P\left(\tau_{0} \geq T_{b}\right)=0
$$

we obtain (2.12). Theorem 2.2 is proved.

\section{Higher Moment of the Dividend Payments}

By the definitions of $M(u, y ; b)$ and $V(u, b)$, we obtain, for $i \in E$,

$$
\begin{aligned}
& M_{1 i}(u, y ; b)=1+\sum_{n=1}^{\infty} \frac{y^{n}}{n !} V_{n, 1, i}(u, b) \\
& M_{2 i}(u, y ; b)=1+\sum_{n=1}^{\infty} \frac{y^{n}}{n !} V_{n, 2, i}(u, b)
\end{aligned}
$$

where $V_{n, i}(u ; b)=\left\{\begin{array}{l}V_{n, 1, i}(u ; b), 0 \leq u<b \\ V_{n, 2, i}(u ; b),-c / \beta<u \leq 0\end{array}\right.$

Substituting (3.1) and (3.2) into (2.1) and (2.2), respectively, and comparing the coefficients of $y^{n}$ yield the following integro-differential equations:

$$
\begin{aligned}
c V_{n, 1, i}^{\prime}(u, b)= & \left(\lambda_{i}+n \delta\right) V_{n, 1, i}(u, b) \\
& -\lambda_{i} \int_{0}^{u} V_{n, 1, i}(u-x, b) \mathrm{d} F_{i}(x) \\
& -\lambda_{i} \int_{u}^{u+\frac{c}{\beta}} V_{n, 2, i}(u-x, b) \mathrm{d} F_{i}(x) \\
& -\sum_{k=1}^{m} \alpha_{i k} V_{n, 1, k}(u, b)
\end{aligned}
$$

for $0 \leq u<b$, and for $-c / \beta<u \leq 0$,

$$
\begin{aligned}
(\beta u+c) V_{n, 2, i}^{\prime}(u, b)= & \left(\lambda_{i}+n \delta\right) V_{n, 2, i}(u, b) \\
& -\lambda_{i} \int_{0}^{u+\frac{c}{\beta}} V_{n, 2, i}(u-x, b) \mathrm{d} F_{i}(x) \\
& -\sum_{k=1}^{m} \alpha_{i k} V_{n, 2, k}(u, b)
\end{aligned}
$$


Substituting (3.1) into (2.3), similarly, we obtain

$$
\left.V_{n, 1, i}^{\prime}(u, b)\right|_{u=b}=n V_{n-1,1, i}(b, b)
$$

thus, $V_{1,1, i}(b, b)=1$ is an obvious result since $V_{0,1, i}(b, b)=1$.

Substituting (3.1) and (3.2) into (2.4) and (2.12), we obtain, for $n \in N^{+}$

$$
\begin{aligned}
& V_{n, 2, i}(-c / \beta, b)=0 \\
& V_{n, 1, i}(0+, b)=V_{n, 2, i}(0-, b)
\end{aligned}
$$

Letting $u \downarrow 0$ in (3.3) and $u \downarrow 0$ in (3.4) and using (3.7), we obtain, for $n \in N^{+}$

$$
V_{n, 1, i}^{\prime}(0+, b)=V_{n, 2, i}^{\prime}(0-, b)
$$

\section{Expected Discounted Penalty Function}

In this section, we derive integro-differential equations for the expected discounted penalty function. For $i \in E$, define

$$
\Phi_{i}(u, b)= \begin{cases}\Phi_{1 i}(u, b), & 0 \leq u<b \\ \Phi_{2 i}(u, b), & -c / \beta<u \leq 0\end{cases}
$$

Theorem 4.1 For $0 \leq u \leq b$,

$$
\begin{aligned}
c \Phi_{1, i}^{\prime}(u, b)= & \left(\lambda_{i}+\delta\right) \Phi_{1, i}(u, b) \\
& -\lambda_{i} \int_{0}^{u} \Phi_{1, i}(u-x, b) \mathrm{d} F_{i}(x) \\
& -\lambda_{i} \int_{u}^{u+\frac{c}{\beta}} \Phi_{2, i}(u-x, b) \mathrm{d} F_{i}(x) \\
& -\sum_{k=1}^{m} \alpha_{i k} \Phi_{1, k}(u, b)-\lambda_{i} A_{i}(u), \quad i \in E
\end{aligned}
$$

and, for $-c / \beta<u \leq 0$,

$$
\begin{aligned}
(\beta u+c) \Phi_{2, i}^{\prime}(u, b)= & \left(\lambda_{i}+\delta\right) \Phi_{2, i}(u, b) \\
& -\lambda_{i} \int_{0}^{u+\frac{c}{\beta}} \Phi_{2, i}(u-x, b) \mathrm{d} F_{i}(x) \\
& -\lambda_{i} A_{i}(u)-\sum_{k=1}^{m} \alpha_{i k} \Phi_{2, i}(u, b), \quad i \in E
\end{aligned}
$$

with boundary conditions $\Phi_{1, i}^{\prime}(b, b)=0$

$$
\begin{aligned}
& \Phi_{1, i}(0+, b)=\Phi_{2, i}(0-, b) \\
& \Phi_{1, i}^{\prime}(0+, b)=\Phi_{2, i}^{\prime}(0-, b)
\end{aligned}
$$

where

$$
A_{i}(u)=\int_{u+\frac{c}{\beta}}^{\infty} \omega(u, x-u) \mathrm{d} F_{i}(x)
$$

Proof. For $i \in E$ and $0 \leq u \leq b$. Similar to argument as in Section 2, we condition on the events that can occur in the small time interval $[0, t]$.

$$
\begin{aligned}
\Phi_{1 i}(u, b)= & \left(1-\alpha_{i} t-\lambda_{i} t\right) \mathrm{e}^{-\delta t} \Phi_{1 i}(u+c t, b) \\
& +\lambda_{i} t \mathrm{e}^{-\delta t} \int_{0}^{u+c t} \Phi_{1 i}(u+c t-x, b) \mathrm{d} F_{i}(x) \\
& +\lambda_{i} t \mathrm{e}^{-\delta t} \int_{u+c t}^{u+c t+\frac{c}{\beta}} \Phi_{2 i}(u+c t-x, b) \mathrm{d} F_{i}(x) \\
& +\lambda_{i} \mathrm{e}^{-\delta t} \int_{u+\frac{c}{\beta}}^{\infty} \omega(u, x-u) \mathrm{d} F_{i}(x) \\
& +t \mathrm{e}^{-\delta t} \sum_{k=1, k \neq i}^{m} \alpha_{i k} \Phi_{1 k}(u+c t, b) \\
& +o(t)
\end{aligned}
$$

Since $\mathrm{e}^{-\delta t}=1-\delta h+o(h)$

we then get

$$
\begin{aligned}
\Phi_{1 i}(u, b)= & {\left[1-\left(\alpha_{i}+\lambda_{i}+\delta\right) t\right] \Phi_{1 i}(u+c t, b) } \\
& +\lambda_{i} t \int_{0}^{u+c t} \Phi_{1 i}(u+c t-x, b) \mathrm{d} F_{i}(x) \\
& +\lambda_{i} t \int_{u+c t}^{u+c t+\frac{c}{\beta}} \Phi_{2 i}(u+c t-x, b) \mathrm{d} F_{i}(x) \\
& +\lambda_{i} t \int_{u+\frac{c}{\beta}}^{\infty} \omega(u, x-u) \mathrm{d} F_{i}(x) \\
& +t \sum_{k=1, k \neq i}^{m} \alpha_{i k} \Phi_{1 k}(u+c t, b)+o(t)
\end{aligned}
$$

Equation (4.7) can be rewritten as

$$
\begin{aligned}
& \frac{\Phi_{1 i}(u+c t, b)-\Phi_{1 i}(u, b)}{t} \\
& =\left(\alpha_{i}+\lambda_{i}+\delta\right) \Phi_{1 i}(u+c t, b) \\
& \quad-\lambda_{i} \int_{0}^{u+c t} \Phi_{1 i}(u+c t-x, b) \mathrm{d} F_{i}(x) \\
& \quad-\lambda_{i} \int_{u+c t+\frac{c}{\beta}}^{u+c t} \Phi_{2 i}(u+c t-x, b) \mathrm{d} F_{i}(x) \\
& \quad-\lambda_{i} \int_{u+\frac{c}{\beta}}^{\infty} \omega(u, x-u) \mathrm{d} F_{i}(x) \\
& \quad-\sum_{k=1, k \neq i}^{m} \alpha_{i k} \Phi_{1 k}(u+c t, b)+\frac{o(t)}{t}
\end{aligned}
$$

Letting $t \rightarrow 0$ in (4.8) and noting that $\alpha_{i i}=-\alpha_{i}$, we obtain (4.1).

For $i \in E$ and $-c / \beta<u \leq 0$, we have

$$
\begin{aligned}
\Phi_{2 i}(u, b)= & \left(1-\alpha_{i} t-\lambda_{i} t\right) \mathrm{e}^{-\delta t} \Phi_{2 i}\left(h_{\beta}(u, b), b\right) \\
& +\lambda_{i} t \mathrm{e}^{-\delta t} \int_{0}^{h_{\beta}(u, t)+\frac{c}{\beta}} \Phi_{2 i}\left(h_{\beta}(u, b)-x, b\right) \mathrm{d} F_{i}(x) \\
& +\lambda_{i} t \mathrm{e}^{-\delta t} \int_{h_{\beta}(u, t)+\frac{c}{\beta}}^{\infty} \omega\left(h_{\beta}(u, b), x-h_{\beta}(u, b)\right) \mathrm{d} F_{i}(x) \\
& +t \mathrm{e}^{-\delta t} \sum_{k=1, k \neq i}^{m} \alpha_{i k} \Phi_{2 k}\left(h_{\beta}(u, b), b\right)+o(t)
\end{aligned}
$$

By Taylor’s expansion 


$$
\Phi_{2 i}\left(h_{\beta}(u, b), b\right)=\Phi_{2 i}(u, b)+(\beta u+c) t \Phi_{2 i}^{\prime}(u, b)+o(t)
$$

Substituting (4.10) into (4.9), dividing both sides by $t$, and letting $t \rightarrow 0$, we obtain (4.2). Theorem 4.1 is proved.

Integro-differential Equations (4.1) and (4.2) can easily be rewritten in matrix form.

Let $\boldsymbol{\Phi}_{j}(u, b)=\left(\Phi_{j 1}(u, b), \cdots, \Phi_{j m}(u, b)\right)^{\mathrm{T}}, j=1,2$.

"T" denoting transpose. Then the vectors of the expected discounted penalty function $\boldsymbol{\Phi}_{1}(u, b)$ and $\boldsymbol{\Phi}_{2}(u, b)$ satisfy the following integro-differential equations

$$
\begin{aligned}
\Phi_{1}^{\prime}(u, b)= & \boldsymbol{P}_{1} \Phi_{1}(u, b) \\
& +\int_{0}^{u} \boldsymbol{G}_{1}(x) \Phi_{1}(u-x, b) \mathrm{d} x \\
& +\int_{u}^{u+\frac{c}{\beta}} \boldsymbol{G}_{1}(x) \Phi_{2}(u-x, b) \mathrm{d} x \\
& +A_{1}(u), 0 \leq u \leq b \\
\Phi_{2}^{\prime}(u, b)= & P_{2}(u) \Phi_{2}(u, b) \\
& +\int_{0}^{u+\frac{c}{\beta}} \boldsymbol{G}_{2}(x) \Phi_{2}(u-x, b) \mathrm{d} x \\
& +\boldsymbol{A}_{2}(u),-c / \beta<u \leq 0
\end{aligned}
$$

where $\quad \boldsymbol{P}_{1}=\left[\operatorname{diag}\left(\lambda_{1}+\delta, \cdots, \lambda_{m}+\delta\right)-\Lambda\right] / c$

$$
\begin{gathered}
\boldsymbol{P}_{2}(u)=\left[\operatorname{diag}\left(\lambda_{1}+\delta, \cdots, \lambda_{m}+\delta\right)-\Lambda\right] /(\beta u+c) \\
\boldsymbol{G}_{1}(x)=-\operatorname{diag}\left(\lambda_{1} f_{1}(x), \cdots, \lambda_{m} f_{m}(x)\right) / c \\
\boldsymbol{G}_{2}(x)=-\operatorname{diag}\left[\frac{\lambda_{1} f_{1}(x)}{(\beta u+c)}, \ldots, \frac{\lambda_{m} f_{m}(x)}{(\beta u+c)}\right]
\end{gathered}
$$

are all $m \times m$ matrices, and $\boldsymbol{A}_{1}(u)$ and $\boldsymbol{A}_{2}(u)$ defined by

$$
\begin{aligned}
& \boldsymbol{A}_{1}(u)=\int_{u+\frac{c}{\beta}}^{\infty} \omega(u, x-u) \boldsymbol{G}_{1}(x) \boldsymbol{I} \mathrm{d} x \\
& \boldsymbol{A}_{2}(u)=\int_{u+\frac{c}{\beta}}^{\infty} \omega(u, x-u) \boldsymbol{G}_{2}(x) \boldsymbol{I} \mathrm{d} x
\end{aligned}
$$

are all $m$-dimensional vector, in which $\boldsymbol{I}=(1,1, \cdots, 1)^{\mathrm{T}}$ is an $m \times 1$ column vector. The continuity condition and derivative condition for $\Phi_{1}(u, b)$ and $\Phi_{2}(u, b)$ is

$$
\begin{aligned}
& \boldsymbol{\Phi}_{1}(0+, b)=\Phi_{2}(0-, b) \\
& \Phi_{1}^{\prime}(0+, b)=\Phi_{2}^{\prime}(0-, b)
\end{aligned}
$$

\section{Time to Reach the Dividend Barrier}

In this section, we consider how long it takes for the surplus process to reach the dividend barrier $b$ from the initial surplus $u$ without ruin occurring. We define $\tau_{b}$ to be the first time that the surplus reaches $b$, and for $\rho>0$, $i \in E$ and $-c / \beta<u \leq b$, define

$$
L_{i}(u, b)=E\left[e^{-\rho \tau_{b}} I\left(\tau_{b}<T\right) \mid U_{b}(0)=u, J(0)=i\right]
$$

$L_{i}(u, b)$ can be interpreted as the expected present value of one dollar payable at the time of reaching the barrier $b$ without ruin occurring, given that the initial environment state is $i$ and the initial surplus is $u$. Alternatively, it can be viewed as the Laplace transform of the time to reach the dividend barrier $b$ without ruin occurring with respect to the parameter $\rho$.

$$
\text { We define } L_{i}(u ; b)= \begin{cases}L_{1, i}(u ; b) & 0 \leq u<b \\ L_{2, i}(u ; b) & -c / \beta<u \leq 0\end{cases}
$$

Using the same arguments as in Section 2, we can easily show that $L_{i}(u, b)$ satisfies the following integrodifferential equations:

$$
\begin{aligned}
c L_{1, i}^{\prime}(u, b)= & \left(\lambda_{i}+\delta\right) L_{1, i}(u, b) \\
& -\lambda_{i} \int_{0}^{u} L_{1, i}(u-x, b) \mathrm{d} F_{i}(x) \\
& -\lambda_{i} \int_{u}^{u+\frac{c}{\beta}} L_{2, i}(u-x, b) \mathrm{d} F_{i}(x) \\
& -\sum_{k=1}^{m} \alpha_{i k} L_{1, k}(u, b)
\end{aligned}
$$

for $0 \leq u<b$, and for $-c / \beta<u \leq 0$,

$$
\begin{aligned}
(\beta u+c) L_{2, i}^{\prime}(u, b)= & \left(\lambda_{i}+\delta\right) L_{2, i}(u, b) \\
& \left.-\lambda_{i} \int_{0}^{u+\frac{c}{\beta}} L_{2, i}(u-x, b)\right) \mathrm{d} F_{i}(x) \\
& -\sum_{k=1}^{m} \alpha_{i k} L_{2, k}(u, b)
\end{aligned}
$$

with boundary conditions

$$
\begin{aligned}
& \left.L_{1, i}(u ; b)\right|_{u=b}=1 \\
& L_{1, i}(0+; b)=L_{2, i}(0-; b) \\
& L_{1, i}^{\prime}(0+; b)=L_{2, i}^{\prime}(0-; b)
\end{aligned}
$$

\section{Acknowledgements}

We would like to thank the anonymous referee who gave us many insightful suggestions and valuable comments on the previous version of this paper. This work is supported by Humanities and Social Sciences Project of the Ministry Education of China (No. 09YJC910004, No. 10YJC630092) and Natural Science Foundation of Shandong Province (No. ZR2010GL013) and Research Program of Higher Education of Shandong Province (No. J10WF84) and Natural Science Foundation of Shandong Jiaotong University (No. Z201031). 


\section{References}

[1] J. M. Reinhard, "On a Class of Semi-Markov Risk Models Obtained as Classical Risk Models in a Markovian Environment,” ASTIN Bulletin, Vol. 14, 1984, pp. 23-43.

[2] S. Asmussen, "Risk Theory in a Markovian Environment," Scandinavian Actuarial Journal, Vol. 2, 1989, pp. 69100.

[3] Y. Lu and S. Li, "On the Probability of Ruin in a Markov-Modulated Risk Model,” Insurance: Mathematics and Economics, Vol. 37, No. 3, 2005, pp. 522-532. doi:10.1016/j.insmatheco.2005.05.006

[4] A. Ng and H. Yang, "Lundberg-Type Bounds for the Joint Distribution of Surplus Immediately before and after Ruin under a Markov-modulated Risk Model,” Astin Bulletin, Vol. 35, 2005, pp. 351-361. doi:10.2143/AST.35.2.2003457

[5] A. Ng and H. Yang, "On the Joint Distribution of Surplus Prior and Immediately after Ruin under a Markovian Regime Switching Model," Stochastic Processes and Their Applications, Vol. 116, No. 2, 2006, pp. 244-266. doi:10.1016/j.spa.2005.09.008

[6] S. M. Li and Y. Lu, "Moments of the Dividend Payments and Related Problems in a Markov-Modulated Risk Model,” North American Actuarial Journal, Vol. 11, No. 2, 2007, pp. 65-76.

[7] Y. Lu and S. Li, "The Markovian Regime-switching Risk Model with a Threshold Dividend Strategy," Insurance: Mathematics and Economics, Vol. 44, No. 2, 2009, pp. 296-303. doi:10.1016/j.insmatheco.2008.04.004

[8] J. Liu, J. C. Xu and H. C. Hu, “The Markov-Dependent
Risk Model with a Threshold Dividend Strategy," Wuhan University Journal of Natural Sciences, Vol. 16, No. 3, 2011, pp. 193-198. doi:10.1007/s11859-011-0736-9

[9] J. Zhu and H. Yang, "Ruin Theory for a Markov Regime-Switching Model under a Threshold Dividend Strategy,” Insurance: Mathematics and Economics, Vol. 42, No. 1, 2008, pp. 311-318. doi:10.1016/j.insmatheco.2007.03.004

[10] J. Q. Wei, H. L. Yang and R. M. Wang, "On the Markov -Modulated Insurance Risk Model with Tax,” Blaetter der DGVFM, Vol. 31, No. 1, 2010, pp. 65-78. doi:10.1007/s11857-010-0104-4

[11] M. Zhou and C. Zhang, “Absolute Ruin under Classical Risk Model,” Acta Mathematicae Applicate Sinica, Vol. 28, No. 4, 2005, pp. 57-80.

[12] J. Cai, "On the Time Value of Absolute Ruin with Debit Interest,” Advances in Applied Probability, Vol. 39, No. 2, 2007, pp. 343-359. doi:10.1239/aap/1183667614

[13] H. L. Yuan and Y. J. Hu, “Absolute Ruin in the Compound Poisson Risk Model with Constant Dividend Barrier," Statistics and Probability Letter, Vol. 78, No. 14, 2008, pp. 2086-2094. doi:10.1016/j.spl.2008.01.076

[14] C. W. Wang and C. C. Yin, "Dividend Payments in the Classical Risk Model under Absolute Ruin with Debit Interest," Applied stochastic models in business and Industry, Vol. 25, No. 3, 2009, pp. 247-262. doi:10.1002/asmb.722

[15] C. W. Wang, C. C. Yin and E. Q. Li, "On the Classical Risk Model with Credit and Debit Interests under Absolute Ruin," Statistics and Probability Letters, Vol. 80, No. 15, 2010, pp. 427-436. doi:10.1016/j.spl.2009.11.020 